\title{
Creación de Grupos de Intervención en Habilidades Sociales y Como Grupo de
}

\author{
Apoyo

\section{Creation of Intervention Groups in Social Skills and as a Support Group}

\author{
Berta Fraga, Leticia Sambade, Beatriz López
}

\author{
Asociación DISMACOR
}

\begin{abstract}
Resumen
Las personas con diversidad funcional y, en concreto, las personas englobadas dentro del Trastorno del Espectro Autista, presentan importantes dificultades para poder establecer unas pautas de relación e interacción adecuadas que les permitan elaborar lazos estables con iguales en su desarrollo. En este trabajo se presenta una propuesta de intervención en habilidades sociales que se basa en la creación de grupos de personas con características comunes que, además de permitir poner en práctica aquellos aspectos sociales deficitarios, se constituirá como grupo de referencia y apoyo que permitirá mejorar el sentimiento de pertenencia y competencia social de los usuarios.

Palabras clave: habilidades sociales, grupos de apoyo, desarrollo social, trastorno del espectro autista.
\end{abstract}

\begin{abstract}
People with disabilities and those who have autism, have important difficulties to set appropriate interactions, which let them to have good relationships. In these lines a new way of intervention is shown, which is based on the creation of groups of people with similar characteristics in which social skills are practiced and which is thought to form a support group that would improve social and personal development.

Keywords: social skills, support groups, social development, autism.
\end{abstract}

\section{Introducción}

El concepto de habilidades sociales se constituye como un constructo difícilmente definible que, sin embargo, ocupa un importante papel en el desarrollo de la persona como tal. En este sentido, la revisión de diferentes definiciones profesionales sobre dicho concepto, arroja la presencia de diversos aspectos comunes (Santos y Lorenzo, 1999) de los que se deriva que las habilidades sociales son una serie de conductas instrumentales aprendidas (Braz, Cômodo, Del Prette, Del Prette y Fontaine, 2013) encaminadas a alcanzar una meta, socialmente aceptadas y adaptadas al contexto determinado de desarrollo de la persona, que se caracterizan por la presencia de aspectos observables y no observables (en concreto, componentes cognitivos y afectivos) y permiten que se lleve a cabo una correcta interacción con los que nos rodean (Iruarrizaga, GómezSegura, Criado, Zuazo y Sastre, 1997).
Diferentes estudios muestran la relación entre las competencias en habilidades sociales y unos niveles óptimos de autoestima y autoconcepto, además de incidir significativamente en el funcionamiento tanto académico como familiar de las personas en todos los periodos de su vida (Rosa, Inglés, Olivares, Espada, Sánchez-Meca y Méndez, 2002) y suponer un factor de riesgo en la aparición de trastornos psicopatológicos y de la conducta social (Betina y Contini, 2011).

La mayor parte de las personas con diversidad funcional y, en concreto, las personas englobadas dentro del Trastorno Autista, tienen problemas relacionadas con la comprensión social, comprensión de las emociones propias y ajenas y dificultades para responder de manera adecuada y coherente a las mismas, alteraciones en el comportamiento no verbal (contacto físico y ocular, postura corporal, respeto del espacio interpersonal...) y la consonancia del mismo con los mensajes lingüísticos emitidos (Pérez, 2016).

Investigaciones recientes relacionan la presencia de estos déficits en las competencias sociales con alteraciones a nivel de cognición social, relacionadas con dificultades para integrar la información que procede de las diferentes fuentes implicadas en el contexto comunicativo (Martos-Pérez, 2008); que pueden ser explicadas por alteraciones a nivel de funcionamiento de la amígdala (Tuchman, 2001) y de los lóbulos frontales, encargados de controlar las funciones ejecutivas, memoria de trabajo, inhibición, resolución de problemas, interacción social, comunicación y relaciones afectivas (Comin, 2013).

Los déficits en habilidades sociales de este tipo de personas suponen implicaciones en el desempeño de situaciones de la vida cotidiana: alteraciones a la hora de acceder al empleo por fallos en la comprensión de situaciones y demandas sociales asociadas, acceso a medios sociosanitarios por fallos en la comprensión de los procedimientos médicos, y aspectos relacionados con la funcionalidad de la vida diaria (tener pareja e hijos, hacer la compra, acudir a eventos sociales...) (Garrido, Carballo, Franco y García-Retamero, 2015; Reilly, McKean, Morgan y Wake, 2016). 


\section{Método}

La Asociación DISMACOR nace con el fuerte propósito de asegurar una participación completa de las personas con diversidad funcional dentro del marco de referencia en el que se desarrollan, procurando, para ello, dotar a todos los usuarios de unas habilidades específicas que les permitan tener unos niveles de calidad de vida aceptables. En este sentido, la metodología interventiva se basa en una atención a domicilio y a la carta que incida en las necesidades específicas y actuales de la persona y de sus familiares; entendiendo que toda intervención en el ambiente natural de la persona favorecerá una mayor generalización de los resultados conseguidos.

\section{Participantes}

La creación del grupo de apoyo e intervención en habilidades sociales parte del conocimiento de los participantes gracias a las sesiones individuales. Así, el grupo está formado por cuatro sujetos de entre ocho y once años, todos ellos diagnosticados con trastorno del espectro autista o características similares, que reciben atención personalizada e individualizada por parte de nuestra asociación desde hace varios años. En concreto podemos diferenciar:

- Un niño de ocho años diagnosticado con Síndrome de Asperger. El niño, que acude a un centro de educación normativa, acaba de terminar tercero de educación primaria con unos resultados académicos excelentes, sin refuerzo académico ni adaptación curricular. Este año ha comenzado a recibir apoyo de pedagogía terapéutica dentro del centro para tratar dificultades a nivel de comprensión y ejecución en contextos sociales. Sus principales dificultades radican en problemas de flexibilidad en relación a gustos e intereses y asociados a la incapacidad para aceptar cambios en sus rutinas diarias o en sus concepciones y pensamientos; ante los cuales reacciona de manera explosiva

- Un niño de diez años diagnosticado de autismo desde los cuatro años. Actualmente ha terminado cuarto de educación primaria (tras haber flexibilizado en el último curso de educación infantil) sin ningún tipo de apoyo ni adaptación curricular. Las principales dificultades del pequeño están relacionadas con problemas para comprender y actuar en consonancia con las situaciones sociales en las que se ve envuelto, cuya complejidad va en aumento constantemente.

- Una niña de once años recién cumplidos diagnosticada de autismo de alto funcionamiento y Trastorno Obsesivo Compulsivo. Actualmente la pequeña acaba de terminar quinto de educación primaria con unos excelentes resultados y sin necesidad de apoyo específico a nivel académico. Sin embargo, las importantes dificultades de interacción y comprensión de la realidad social implican la necesidad de contar con una persona de apoyo en los momentos de recreo, salidas, excursiones y deambulación por el centro, que eviten que la niña se aísle de manera acusada y no participe de las actividades grupales de la institución; lo que dificulta la participación como miembro de pleno derecho de la realidad sociocultural del centro. Además, estas dificultades de comprensión suponen importantes afectaciones en el nivel de ansiedad de la pequeña (que, actualmente, es medicada para ello).

- Un niño de once años con claros problemas de atención, impulsividad, dificultades de comprensión de aspectos sociales, alteraciones en el lenguaje, inflexibilidad y presencia de intereses absorbentes. A pesar de estas dificultades, no se ha cumplido el criterio clínico significativo que pueda identificar al niño con alguna categoría diagnóstica. Se ha probado medicación para tratar los déficits de atención, observándose un empeoramiento acusado de la capacidad funcional del pequeño, por lo que se decide retirar. Actualmente el niño ha terminado la primaria con buenos resultados académicos, aun con necesidad de pequeños refuerzos en casa.

Tabla 1.

Características de los participantes

\begin{tabular}{lccc}
\hline Sujeto & Sexo & Edad & Diagnóstico \\
\hline 1 & Hombre & 8 & Síndrome de Asperger \\
2 & Hombre & 10 & $\begin{array}{c}\text { Autismo } \\
3\end{array}$ \\
Mujer & 11 & Autismo de alto funcionamiento \\
TOC \\
4 & Hombre & 11 & $\begin{array}{c}\text { Déficit de atención } \\
\text { Trastorno del Lenguaje } \\
\text { Inflexibilidad e intereses } \\
\text { absorventes }\end{array}$ \\
\hline
\end{tabular}

Por tanto, el grupo tiene como característica común la necesidad de trabajar las habilidades sociales de cara a mejorar su funcionalidad en la interacción con el entorno, lo que redundará significativamente en su calidad de vida.

\section{Objetivos}

El objetivo general de este tipo de intervención radica en la instauración de unas capacidades adecuadas en habilidades sociales que permitan que el niño obtenga un funcionamiento pleno que les permita desarrollarse adecuadamente en el mundo al que pertenecen. De la misma manera, se busca que los niños establezcan unos lazos relativamente estables con los demás participantes que les permitan contar con un grupo de apoyo tan necesario en todas las etapas de la vida de la persona, pero especialmente en las edades de las que estamos hablando. Estos objetivos generales se especifican en los siguientes objetivos específicos:

- Mejorar la capacidad de escucha activa y participación en conversaciones de manera recíproca. Se observa que todos los participantes tienen tendencia a no escuchar lo que les comunica el interlocutor, por lo que no son capaces de responder de manera adecuada a lo que se les formula $y$, finalmente, tienden a hablar mediante monólogos sobre sus temas fetiches sin tener en 
cuenta la adecuación de los mismos a la situación contextual o a los intereses de quienes les rodean.

- Incrementar la competencia de narrar experiencias vividas. En general, los discursos de los niños se caracterizan por la ausencia de cohesión entre las ideas que se quieren mostrar que, además, suelen ser expresadas de manera incompleta; por lo que suele ser necesario que el receptor sepa de qué tema está hablando el niño para poder comprender el sentido del mensaje.

- Desarrollar comportamientos prosociales que permitan establecer unos niveles de empatía que se encuentren en la base del establecimiento de relaciones sociales de calidad. En general, los niños tienden a pasar por alto indicadores (tanto verbales como no verbales) que muestran las necesidades del interlocutor; de esta manera, puede que muchas de sus respuestas no sean consecuentes con el contexto social en el que se esté desarrollando la situación comunicativa.

- Reconocimiento de los propios sentimientos y desarrollo de las competencias necesarias para poder comunicarlos y hacer que sean respetados por los demás.

- Reducción de comportamientos y conductas disruptivas que aparecen cuando no son capaces de comunicar sus sentimientos de manera efectiva o de hacer valer sus pensamientos, especialmente cuando se refiere a alguna cuestión en la que no estén de acuerdo.

- Favorecer competencias funcionales de toma de decisiones y solución de problemas, que impliquen procedimientos democráticos y respetuosos para todos los miembros.

- Desarrollo de una visión realista sobre sí mismos, sus capacidades, puntos fuertes y debilidades. En general, se observa que los niños tienden a tener una visión no realista sobre su propio yo (en algunos casos por ser demasiado positiva y en otras, negativa).

- Mejorar la autonomía en su interacción en la vida diaria. Son pocas las actividades que realizan sin la supervisión de algún adulto cercano.

\section{Instrumentos}

Para la puesta en marcha de las sesiones se utilizan diversos materiales propios del entrenamiento en actividades grupales, así como manuales y ejercicios específicos para cada apartado trabajado.

De cara a obtener información objetiva que nos permita valorar los resultados obtenidos con la metodología propuesta, se realiza un procedimiento de observación pormenorizada de las situaciones de interacción (tanto dirigida como libre) de los usuarios, analizando aquellos aspectos de la interacción y el funcionamiento social que se han estado trabajando mediante la intervención grupal.

De la misma manera, se realizan entrevistas a los padres y a los propios niños para poder valorar el grado de adecuación de los resultados obtenidos con los objetivos planteados en el programa de intervención.

\section{Procedimiento}

Se reúne a los niños en momentos lúdicos, intentando que se corresponda con momentos de vacaciones del colegio, o fines de semana. Este tipo de intervención comenzó el verano pasado, como consecuencia de observar la necesidad de trabajar aspectos de interacción que no podían ser atendidos mediante intervención individual.

Para ello, tras la conformidad con los progenitores, se establecen una cadencia de intervención que será de dos horas semanales en los periodos vacacionales y dos horas mensuales durante el resto del curso.

Las sesiones se organizarán en diferentes apartados que se repetirán de manera periódica, constatando los siguientes componentes:

1. Saludo y puesta al día: los niños y el terapeuta se reúnen en torno a una mesa. Una de las habilidades que pretendemos potenciar con este tipo de intervención, es la capacidad de escucha y conversación, por lo que es importante que los niños aprendan a escuchar y contar experiencias vividas, interesándose por las de los demás con preguntas relacionadas. Para ello, cada participante contará algo relevante que le haya ocurrido durante el periodo de tiempo en el que no se han visto; el terapeuta también realizará el ejercicio para servir de modelo.

2. Ejercicios propios de entrenamiento en habilidades sociales, dentro de estos destacamos un programa de intervención que tiene en cuenta los siguientes aspectos:

- Entrenamiento en comportamiento no verbal, tanto para su correcta implementación como para el reconocimiento y comprensión del mismo, que les ayude a tener un mejor funcionamiento en las situaciones sociales en las que se desarrollen.

- Entrenamiento en asertividad, siendo capaces de comunicar sus sentimientos y deseos sin herir al receptor, pero sin dejar de reclamar sus derechos. De la misma manera, se trabaja la comunicación de sentimientos y respuestas negativas de manera asertiva.

- Escucha activa, ejercicios encaminados a comprender y dar a entender que estamos comprendiendo e interesados por lo que el otro nos cuenta.

- Entrenamiento en solución de problemas, aprendiendo a identificar el problema que se les plantea, las posibles soluciones alternativas $\mathrm{y}$, finalmente, elegir la que más se adecúa a las necesidades del momento.

3. Ejercicios grupales para trabajar aspectos personales indispensables en la formación del niño como agente completo. Así, se tratan temas como el autoconcepto (aceptación de aspectos tanto físicos como de personalidad), autoestima, autoeficacia, valores... 
4. Participación en situaciones cotidianas de la vida diaria que impliquen interacción con otros agentes y cierta dosis de autonomía. Normalmente se lleva a los niños a comprar (siendo ellos los que decidan, en grupo, qué deben comprar y si les llega el dinero que tienen), a cafeterías o a realizar actividades lúdicas en las que deban hablar con monitores o encargados desconocidos.

5. Se les insta a que decidan entre todos una actividad lúdica en la que todos los miembros participen, siendo valorados y respetados.

6. Para finalizar, deben acompañar a sus compañeros a sus casas y despedirse de los mismos.

Se postula una intervención grupal de cara a asegurar un modelado por parte de los miembros hacia el resto de compañeros, además de ser un medio para estimular la creatividad y flexibilidad a la hora de proponer alternativas y posibilidades; aspecto que, como hemos señalado, se muestra alterado en todos los sujetos que forman parte del programa.

\section{Resultados}

La puesta en marcha de esta línea de intervención ha mostrado cambios sustanciales en el comportamiento social e interactivo de los niños que forman parte de la misma.

De esta manera, los resultados obtenidos mediante observación directa y entrevistas, nos muestran un incremento en la emisión de respuestas prosociales, un aumento del uso de respuestas tanto verbales como no verbales para demostrar interés por lo que los demás nos están contando; además de evidenciar un aumento en la flexibilidad de los temas que les interesan. En esta misma línea se observa una tendencia por ir incorporando los temas fetiches de los demás, o por lo menos elementos de los mismos, a las líneas de interés propias.

A nivel de resolución de problemas y toma de decisiones se ha comprobado que se comienzan a utilizar métodos democráticos de manera más autónoma (ya no es necesario que el terapeuta les inste a ello en todas las ocasiones) y, comienzan a ser capaces de comunicar aquello que les resulta desagradable con unas respuestas más o menos aceptables. Si bien debemos destacar que la impulsividad sigue bastante presente en la mayor parte de ellos, por lo que son comunes los berrinches cuando hay algo que se escapa de sus expectativas.

En cuanto a la repercusión en la vida diaria, se obtienen evidencias (tanto observadas directamente como en boca de sus familiares) de que los niños comienzan a ganar y demandar autonomía en actividades que anteriormente no realizaban sin la ayuda de sus padres.

Finalmente, se observa una mayor implicación de los niños dentro del grupo; constatando una mejor relación entre dos parejas por similitud de caracteres. Además, los niños reclaman realizar actividades y sesiones conjuntas cada vez con mayor frecuencia; notándose, de la misma manera, una mayor complicidad cuando están juntos.

\section{Discusión}

Los resultados concuerdan con la hipótesis de partida que postulaba una relación positiva entre el entrenamiento en habilidades sociales y un incremento en la funcionalidad de su día a día. De esta manera, en los indicado por Rosa y cols. (2002), la competencia en habilidades sociales incide directamente en el desarrollo familiar en tanto que son los propios padres los que informan de una mejoría en el comportamiento y actitudes de sus hijos a la hora de relacionarse, comprender y responder a situaciones sociales dentro del ámbito cotidiano $\mathrm{y}$, en concreto, informan de una reducción de los comportamientos disruptivos y un aumento de respuestas asertivas que permiten comprender los sentimientos que experimentan y sus deseos.

Así mismo, la línea de intervención refuta la idea demostrada por Pérez (2016) de que la intervención en habilidades sociales y la participación en grupos de apoyo suponen un importante referente como constituyente del autoconcepto de la persona, permitiendo que se sienta parte de un conjunto que lo acepta y valora por lo que es.

En el mismo sentido, es importante señalar que los resultados concuerdan con las aportaciones de Garrido y cols. (2015) que señalan el entrenamiento en habilidades sociales como un importante predictor de un mejor desempeño en la vida diaria, en aquellas actividades que implican que los niños se puedan llegar a desarrollar como agentes autónomos e independientes y de pleno derecho; reduciendo, por tanto, en la línea de lo expresado por Betina y cols. (2011), la posibilidad de que desarrollen en un futuro alteraciones psicológicas o problemas de comportamiento prosocial (delincuencia, abandono de estudios, peleas...).

\section{Referencias}

Betina, A. \& Contini, N. (2011). Las Habilidades Sociales en Niños y Adolescentes. Su Importancia en la Prevención de Trastornos Psicopatológicos. Fundamentos en Humanidades, 1(23): 159-182

Braz, A. C., Cômodo, C. N., Del Prette, Z., Del Prette, A. \& Fontaine, A. M. G. (2013). Habilidades Sociales e Intergeneracionalidad en las Relaciones Familiares. Apuntes de Psicología, 31(1).

Comin, D. (2013). El Déficit en la Función Ejectuvia y su Impacto en el Autismo [Mensaje en un blog]. Recuperado de http://autismodiario.org/2013/12/20/eldeficit-en-la-funcionejecutiva-y-su-impacto-enelautismo/

Garrido, D., Carballo, G., Franco, V. \& GarcíaRetamero, R. (2015). Dificultades de Comprensión del Lenguaje en Niños No Verbales con Trastornos del Espectro Autista y sus Implicaciones en la Calidad de Vida Familiar. Revista Neurol, 60 (5): 207-214.

Iruarrizaga, I., Gómez-Segura, J., Criado, T., Zuazo, M. \& Sastre, E. (1997). Reducción de la Ansiedad a Través del Entrenamiento en Habilidades Sociales. Revista Electrónica de Motivación y Emoción, 2 (1).

Martos-Pérez, J. (2005). Intervención Educativa en Autismo desde una Perspectiva Psicológica. Revista Neurol 40(1): 177-180.

Pérez, V. (2016). Los Sistemas Alternativos de Comunicación Como Intervención Comunicativa 
Sobre los Problemas de Conducta en el Trastorno Autista. [TFG]. Universidad de Palermo.

Reilly, S., McKean, C., Morgan, A. \& Wake, M. (2016). La Fluidez, Manejo de los Trastornos del Lenguaje y el Habla en la Infancia. Revista Digital de Ciencias Aplicadas al Deporte, 8 (17).

Rosa, A. I., Inglés, C. I., Olivares, J., Espada, J. P., Sánchez-Meca, J. \& Méndez, X. (2002). Eficacia del Entrenamiento en Habilidades Sociales con Adolescentes: de Menos a Más. Psicología Conductual, 10(3): 543-561.

Santos, M. A., \& Lorenzo, M. M. (1999). La vía comunitaria en las instituciones cerradas: Un programa educativo de habilidades sociales. Revista De Ciencias De La Educación, 177, 79-95.

Tuchman, R.F. (2001). Como Construir un Cerebro Social: lo que nos Enseña el Autismo. Revista de Neurología, 1: 20-33. 